\title{
Stem cells in neurology - current perspectives
}

\author{
Células-tronco em neurologia - perspectivas atuais \\ Chary Ely Marquez Batista ${ }^{1}$, Eric Domingos Mariano ${ }^{1}$, Suely Kazue Nagahashi Marie ${ }^{1}$, Manoel \\ Jacobsen Teixeira ${ }^{1}$, Matthias Morgalla', Marcos Tatagiba ${ }^{2}$, Jun Li', Guilherme Lepski, ${ }^{1,2}$
}

\begin{abstract}
Central nervous system (CNS) restoration is an important clinical challenge and stem cell transplantation has been considered a promising therapeutic option for many neurological diseases. Objective: The present review aims to briefly describe stem cell biology, as well as to outline the clinical application of stem cells in the treatment of diseases of the CNS. Method: Literature review of animal and human clinical experimental trials, using the following key words: "stem cell", "neurogenesis", "Parkinson", "Huntington", "amyotrophic lateral sclerosis", "traumatic brain injury”, "spinal cord injury”, "ischemic stroke”, and "demyelinating diseases”. Conclusion: Major recent advances in stem cell research have brought us several steps closer to their effective clinical application, which aims to develop efficient ways of regenerating the damaged CNS.
\end{abstract}

Keywords: stem cell, neurogenesis, neurological diseases, stem cell therapy.

\section{RESUMO}

Restauração do sistema nervoso central (SNC) é um importante desafio clínico e o transplante de células-tronco tem sido considerado uma opção terapêutica promissora para muitas doenças neurológicas. Objetivo: O presente trabalho tem como objetivo descrever brevemente a biologia das células-tronco, assim como sua aplicação clínica no tratamento de doenças do SNC. Método: Revisão da literatura de experimentação animal e ensaios clínicos com humanos, usando as seguintes palavras chave: "células-tronco", "neurogênese", "Parkinson”, "Huntington”, "esclerose lateral amiotrófica”, “lesão cerebral traumática”, "lesão da medula espinal”, "acidente vascular cerebral isquêmico" e "doenças desmielinizantes". Conclusão: Grandes avanços em pesquisas com células-tronco nos conduziram a novas perspectivas para uma aplicação clínica efetiva, visando desenvolver formas eficazes de regeneração do SNC.

Palavras-chave: células-tronco, neurogênese, doenças neurológicas, terapia com células-tronco.

The central nervous system (CNS) is responsible for the control and coordination of organs and systems in the human body, as well as for integrating the organism with its environment, interpreting the stimuli it receives and generating appropriate responses. This system is made up of noble and highly specialized structures, whose damage may result in serious physical and mental repercussions.

It was previously believed that the adult CNS was fixed and immutable, unable to generate new neurons. In the early twentieth century, Santiago Ramón y Cajal published his work on the regeneration of the nervous system following injury ${ }^{1}$. More recent scientific and technological advancements have shown that the CNS has a considerable regenerative capacity; however, this capacity is not strong enough to single-handedly induce full neuroregeneration.

Several strategies have been developed in an attempt to restore the CNS, including cell therapy, which has shown promise. The development and organization of stem cells is a dynamic and functional process, and its peculiar characteristics, described below, make these cells unique, with great potential for therapeutic applications.

\section{NEURAL STEM/PROGENITORS CELLS}

Stem cells are immature cells with the capacity for selfrenewal, i.e., they are able to proliferate extensively through cell division, give rise to two identical daughter cells, and differentiate into several types of mature and specialized cells. These cells can be found in all multicellular organisms and are responsible for the growth, repair and homeostasis of various tissues ${ }^{2}$.

Stem cells are classified into embryonic stem cells (ESCs), fetal stem cells (FSCs) or adult stem cells (ASCs), depending

${ }^{1}$ Departamento de Neurologia, Faculdade de Medicina, Universidade de São Paulo, Sao Paulo SP, Brazil;

${ }^{2}$ Department of Neurosurgery, Eberhard-Karls University, Tuebingen, Germany.

Correspondence: Guilherme A. Lepski; Av. Dr. Arnaldo, 455 / LIM 15 / $4^{\circ}$ andar; 01246-903 Cerqueira César, São Paulo SP, Brasil; E-mail: lepski@usp.br Conflict of interest: There is no conflict of interest to declare.

Received 16 December 2013; Received in final form 27 March 2014; Accepted 17 April 2014. 
on their origin. They may also be categorized according to their degree of plasticity (i.e., their potential to differentiate into various tissues), as follows: (1) totipotent, (2) pluripotent, or (3) multipotent. Totipotent cells include the zygote and their first progeny cells. These cells are able to generate all types of cells, including embryonic and extra-embryonic tissues. Pluripotent cells are found in the inner cell mass of the blastocyst, and are also known as embryonic stem cells. These cells have the ability to generate cells of all three germ layers: ectoderm, mesoderm and endoderm. Finally, multipotent cells are cells that make up the three germ layers. They are capable of generating different cell types of the tissue in which they reside, are present during embryonic life and, in some tissues, remain in the adult organism. They participate in tissue repair and regeneration, and include mesenchymal stem cells, neural stem cells, bone marrow stromal cells, and olfactory ensheathing cells ${ }^{3}$.

A new line of stem cells known as induced pluripotent stem cells (iPSC) has recently been the focus of intense research. These cells are believed to have the same characteristics as pluripotent ESCs, and are derived from genetically-reprogrammed adult somatic cells. These cells have the potential to produce human iPS from biopsies of any mature somatic tissue, thus generating patient-specific compatible cells. An advantage of iPSCs is that they are exempt from the ethical concerns regarding the use of human embryos. Although results using these cells so far have been promising, some limitations must be overcome before using iPSCs in regenerative medicine, e.g., possible teratoma formation ${ }^{4}$.

Stem cell performance is regulated by the interaction between intrinsic factors and extrinsic signals from the surrounding microenvironment, which is known as the stem cell niche. The stem cell niche is a limited and specialized anatomic compartment where the stem cells reside, and is formed by cellular and molecular components that integrate local and systemic factors, thereby regulating cell proliferation, differentiation and survival. The niche components, as well as the detailed mechanisms underlying their function, are extremely varied. These components include: the stem cell itself; support stromal cells that directly interact with the stem cells through cell surface receptors; gap junctions and soluble factors; extracellular matrix proteins that provide structure, organization and mechanical signals to the niche; blood vessels that carry systemic signs and provide a channel for the recruitment of inflammatory and other circulating cells to the niche; and neural inputs that can communicate distant physiologic signals to the stem cell microenvironment ${ }^{5}$.

ASCs usually remain in a quiescent or low proliferation state and play an important role in tissue homeostasis. They are activated following external injury, leaving their niche to regenerate lost tissue. To maintain the critical balance between stem cell populations and differentiating cells, stem cells have the potential to divide asymmetrically; in other words, one daughter cell remains in the niche as a stem cell and the other daughter cell leaves the niche to differentiate, eventually becoming a functionally mature cell ${ }^{6}$. In the CNS, neural stem cells (NSCs) and their progenitors are commonly referred to as neural precursor cells $(\mathrm{NPCs})^{7}$. NSCs are self-renewing and have the capacity to differentiate into the three neural lineages: neurons, astrocytes and oligodendrocytes. They can be found in the embryonic, neonatal and adult CNS, and participate in the maturation and maintenance of the physiological integrity of this system ${ }^{8}$.

\section{IN VIVO NEUROGENESIS: LOCALIZATION AND FUNCTION}

The process of neurogenesis, which consists in the formation of new neurons from NPCs, normally occurs throughout the brain during prenatal life, and in two major brain regions during adulthood: within the subventricular zone (SVZ) of the frontal horns of the lateral ventricles and in the antero-lateral region of the subgranular layer of the hippocampal dentate gyrus (DG). In these regions, a special type of star-shaped astrocyte (double positive for glial fibrillary acidic protein (GFAP) and nestin), is considered the true physiological NSC. These astrocytes divide asymmetrically at a low duplication rate, producing a cell resembling itself and another small rounded cell, known as a transient amplifying cell (TAC). TACs divide at a very high rate, producing neuroblasts (neural progenitors), and migrate to their definitive places in the CNS while differentiating. In the mature CNS, these new neurons are integrated into the pre-existing neuronal circuitry and take on various functions, thereby contributing to the system's structural and functional plasticity ${ }^{9}$. It is commonly believed that neurogenesis is very limited in other regions of the adult CNS under normal physiological conditions. However, it can be induced after injury ${ }^{10}$.

Adult neurogenesis is a dynamic and functional process. Several intrinsic as well as extrinsic factors can regulate the performance of NSCs, as well as the different stages of their development. These factors include 1) signaling molecules such as Notch, bone morphogenetic protein (BMP), Wnt, sonic hedgehog (Shh) and cAMP response element binding protein (CREB); 2) neurotrophic and growth factors, such as brain-derived neurotrophic factor (BDNF), neurotrophin-3 (NT-3), epidermal growth factor (EGF) and basic fibroblast growth factor (bFGF), and 3) neurotransmitters, such as GABA ( $\gamma$-aminobutyric acid) and glutamate ${ }^{11,12}$.

Different types of physiological or pathological stimuli can interfere with the process of neurogenesis by increasing 
or decreasing the production of new neurons in the adult individual. For example, rats raised in an enriched environment with different stimulating objects or a running wheel show increased production of new neurons in the DG, which stimulates learning ${ }^{13}$. Similarly, the exposure of adult mice to an environment with complex odors increases cells' survival rate as well as the integration of newborn neurons in the olfactory bulb, thus improving olfactory memory ${ }^{14}$. It is interesting to note that adult female rats produce more cells in the DG than their male counterparts ${ }^{15}$. Furthermore, various pathological conditions such as trauma, ischemia and neurodegenerative diseases, can affect neurogenesis in the adult brain. Stem cells and neural progenitors have the intrinsic ability to respond to such insults, increasing their proliferation and generating neurons that appear in the ischemic penumbra surrounding the affected area. This spontaneous response of the adult brain indicates its regenerative potential, but NSCs are not able to induce full neuroregeneration on their own ${ }^{16}$. Other factors that may influence adult neurogenesis by decreasing the production of new hippocampal cells are aging ${ }^{17}$, opioids ${ }^{18}$, stress and depression ${ }^{19}$.

Understanding the mechanisms involved in the generation of new neurons in the adult animal is of great importance. If it were possible to accelerate or stimulate this process, we might be able to promote neurorestoration without the need for biological implants.

\section{TRANSPLANTATION OF EXOGENOUS STEM CELLS}

\section{Stem cells in degenerative diseases \\ Parkinson's disease}

In 1817, British surgeon James Parkinson published a detailed description of a disease which French neurologist Jean Martin Charcot would later call "Parkinson's disease" (PD); distinguished from other neurological diseases characterized by shaking, e.g., multiple sclerosis ${ }^{20}$. PD is a progressive, hypokinetic, and neurodegenerative disease, characterized by motor symptoms such as bradykinesia, rigidity, akinesia, abnormal posture, and residual shaking at rest. It is associated with the loss of dopaminergic neurons (DA) from the substantia nigra (SN). There is some evidence that alterations of several metabolic pathways may cause chronic "neuronal suffering" (i.e., when neurons work harder to make up for cellular death), but the pathogenic sequence of events that leads to DA loss is still unknown ${ }^{21}$.

In recent years, work with humans has grown exponentially. In two important studies by Freed et al. ${ }^{22}$ and Olanow et al. $^{23}$, fetal mesencephalic tissue containing dopamine neurons was transplanted into the putamen of patients. Important questions to consider within this line of work include: the amount of tissue needed to have efficient results, the region of transplant, the local immunologic influence, and the side effects that may appear during the period off medication. In their 2005 review, Winkler et al. ${ }^{24}$ address these questions and reinforce the importance of serotoninergic neurons, which can induce a hyperinervation of the implant. This hyperinervation ensures functional activity and generates sites for the storage and release of dopamine (formed by levodopa, or L-dopa), thus reducing the pharmacological effects of the drug and consequently reducing the risk of dyskinesia. Further work is required to fully understand the safety and efficacy of transplanting fetal tissue containing dopamine cells. It is clear that this type of transplant is an optimal therapeutic strategy for the treatment of Parkinson's disease; however, it is also known that the affected areas go beyond the putamen, so this type of transplantation must be tailored to each patient. It is difficult to establish a safe therapeutic technique for Parkinson's disease because the trigger mechanisms of PD are still unknown. Currently, we know that it is not simply a loss of dopaminergic cells, but a combination of several factors, such as a genetic predisposition, a local neuroinflammatory condition, the influence of protein aggregates, and the participation of other neural cells in the degeneration of dopaminergic neurons ${ }^{25}$.

In addition to the selected treatment, the basic criteria that must be met prior to starting a new clinical trial include the following: developing further in vitro assays, transplanting contaminant-free cells derived from animal cells, recruiting the appropriate patients, and determining regulatory standards. Stem cell transplantation is a promising choice for the treatment of neurological diseases, and gaining a deeper understanding of disease mechanisms and the neurorestorative capacity of these cells is essential for the future of cell transplantation, cultivation and expansion ${ }^{26}$. According to Lindvall and Kokaia, investigators should focus on 3 main tasks: 1) generate large numbers of dopaminergic neurons or neuroblasts in standardized preparations; 2) improve graft efficacy, and 3) minimize the risk of tumor formation and off-medication dyskinesias to start new clinical trials ${ }^{27}$.

\section{Huntington's disease}

Huntington's disease has a genetic, hereditary and dominant character; it is caused by a mutation in the IT15 gene on the short arm of chromosome 4 (4p16.3). It is a lethal progressive disease, characterized by the triad of movement disorders (chorea, dystonia, myoclonus and parkinsonism), behavioral disorders and dementia. The average age of onset is 40-50 years old. Chorea comes on slowly and gradually over a period of several years until it becomes evident. In late/classic manifestations, it involves the face, trunk and limbs. Parkinsonism and dystonia predominate in the earliest manifestations of the disease. Cicchetti et al. ${ }^{28}$ performed 
autopsies on patients who underwent transplantation of fetal NSCs. These authors observed that 2 years after surgery, patients who underwent transplants did not show significant activity. In other words, the presence of an implant did not correlate significantly with patients' improvement. In fact, the immune response seemed to have impaired the functionality of these grafts and patients developed dementia at least 5-years before dying. The transplanted cells remained functionally intact whether or not Lewy bodies were included.

Another issue to consider is how long a transplant may be clinically effective, as it has also been observed that transplanted neurons undergo apoptosis even when they do not contain huntingtin aggregates that are different from the surrounding host tissue ${ }^{29}$. In a histological study performed by Capetian et al. $^{30}$, a probable maturation pattern was observed in the graft. The nucleus was composed of immature and highly proliferative cells that produced a supply of migratory cells; these cells in turn, after crossing the grafthost barrier, migrated to other regions; after they traveled some distance, differentiation took place. Another point to consider is that striata neurons that have not degenerated can reinnervate some reconstituted neurons and thus restore the defective parts of the neuronal network ${ }^{30}$.

There are several open questions regarding neural tissue or stem cell transplantation. Some authors believe that using combined techniques slows down disease progression and promotes neuronal and tissue regeneration ${ }^{31}$.

\section{Amyotrophic lateral sclerosis}

Amyotrophic lateral sclerosis (ALS) is the most common motor neuron disease in adults. It is also known as Charcot disease, as it was first described by Jean-Martin Charcot in 1869. Currently, there are two classifications for ALS Familial ALS (FALS) and sporadic ALS (SALS) - which are clinically similar and are caused by genetic mutations in SOD1, UBQLN2, VCP/p97, TDP-43, FUS/TLS and C9ORF72. ALS begins focally and spreads contiguously. It may first appear in the hands and/or legs, or as dysarthria and/or dyspnea, and then spreads to other body regions. It affects the lower motor neurons of the ventral horn of the spinal cord and the motor nuclei in the brainstem (such as the superior motor cortex) causing an axonal connection failure. These heterogenic mutations alter the cellular physiology of nerve cells and the microenvironment, resulting in excess glutamate, astrocytes and microglial dysfunction, as well as mitochondrial disorders and protein degradation deficits that become natural barriers for therapies ${ }^{32}$.

Due to several of the issues presented in this review, some researchers are not confident about the benefits of autologous stem cell transplantation in patients with ALS. The main concern is the extent to which these cells have been affected by gene mutations. Some groups around the world have conducted clinical trials in humans and have obtained encouraging and surprising results with MSCs; others have opted for using trophic factors to enhance the action of stem cells in preventing neural degeneration (i.e., the neuroprotection theory), rather than properly replacing the lost neurons. Yet another technique involves genetically manipulating neural precursors to overcome the limitations associated with differentiation into motor neurons ${ }^{33}$.

In 2012, Riley and colleagues ${ }^{34}$ published a Phase I trial in which they implanted neural fetal cells at the lumbar level in 12 patients. The group reported no problems with the transplants, thus confirming the safety of this transplantation technique. The existing techniques have had outstanding results in animal models, and a few clinical trials published so far have yielded promising results with humans, but there is still much work to be done to establish a reliable and effective clinical translation of this line of work.

\section{TRAUMATIC BRAIN INJURY (TBI)}

Acute neuropathological conditions such as TBI are a significant cause of death and disability around the world, second only to cancer and cardiovascular disease in Europe and the United States ${ }^{35}$. Diseases where there is loss of one or a few cell types seem to be more responsive to cell transplantation. It is unclear whether the generation of new neurons contributes to the restoration of functions, axonal sprouting, dendritic arborization, synaptic plasticity or neosynaptogenesis. It is known that neurogenesis is restricted to two specific regions during adulthood, and after a TBI, the rate at which these new neurons are generated and functionally integrated is quite low ${ }^{36}$.

As mentioned above, much of what is known about cell transplantation is due to observations from previous studies in patients with Parkinson's and Huntington's disease, which have shown some positive (even if somewhat limited) results. This indicates that future studies will involve transplantation of NPCs and NSCs expanded in culture.

Although the primary focus of their study on TBI was neuronal restoration, Shear et al. ${ }^{37}$ showed that 1 year after NPC transplantation into mice striatum, the animals showed a high capacity for long-term survival and motor improvement, but no tumor formation. However, histochemical analyses revealed no presence of neuronal markers, only oligodendroglial markers. By contrast, when NPS transplantation is made into uninjured brains, one can observe the differentiation into functional mature neurons ${ }^{38}$. Furthermore, immortalized NSCs derived from fetuses secreting GDNF and transplanted into peri-injured cortex trigger improvement in motor functions but no cognitive improvement $^{39}$. When transplanted into the hippocampus, on the other hand, cognitive improvement is observed, but 
no motor improvement. This is probably influenced by changes in the time of transplantation and the number of transplanted cells ${ }^{40}$. In another study, the transplantation of ESCs pre-differentiated into neuronal and glial precursors resulted in great sensory and motor improvements, but no cognitive improvement ${ }^{41}$, whereas in a similar study with GABAergic neuron transplantation, a greater sensorimotor recovery was observed, which was not seen when astrocytes were transplanted ${ }^{42}$. In yet another study, Gao et al. ${ }^{43}$ showed that human fetal NSCs pre-differentiated into neurons and then implanted into the hippocampus of TBI animals, were able to differentiate into neurons 1-day after injury, and the animals showed cognitive improvement. An interesting finding was that some transplanted cells in all animals migrated to the DG, which was explained by the theory that the DG attracts transplanted cells and aids in their differentiation ${ }^{43}$. These findings and those of Gaillard ${ }^{44}$ suggest that the region of the implant appears to exert great influence on cell differentiation; however, the specificity of the transplanted tissue seems to have an even greater influence on functional recovery, because the transplanted cells are capable of capturing cues that influence their differentiation and maturation, cues that may be present in the transplanted region or released after TBI.

NSCs derived from SVZ, expanded in vitro and pre-differentiated into neurons, maintained their neuronal fate and differentiated into functional mature neurons after being transplanted into rat hippocampus. This was confirmed through electrophysiological tests and the presence of GABAergic and glutamatergic synapses. This finding supports the theory that the activity of transplanted neurons can be regulated by host tissue neurons ${ }^{12}$.

In two studies published in 2005 and 2006, Zhu and colleagues ${ }^{45,46}$ transplanted NSCs in patients with TBI. In both studies, the transplanted cells migrated from the region of implant to the periphery of the lesion. Furthermore, when the experimental group began to show increased recovery latency relative to the control group, functional magnetic resonance (fMRI) imaging showed increased activity in areas damaged by injury, positron emission tomography (PET) showed significant improvement in transplanted patients, and somatosensory evoked potentials (SEP) showed no significant improvement until 6 months after transplantation. The experimental group showed rapid recovery beginning at month 6 following transplantation, as measured on the DRS (Disability Rating Scale).

Another factor influencing stem cell transplantation is the route of administration. Intra-arterial MSC administration can cross the blood-brain-barrier, migrate to the border of the injured area, survive and differentiate ${ }^{47}$. In a study conducted by Lu et al., MSCs were found in large numbers 14-days post-transplant. Once again, they were found mostly in the border zone of the injured area, which according to the authors, occurs because the regulation of adhesion molecules can be chemotactic for MSCs. Some motor improvement was observed, yet it was not directly attributed to the MSCs, but to the ability of MSCs to stimulate glial cells to secrete neurotrophic factors, thus decreasing host cell apoptosis and stimulating plasticity ${ }^{48}$.

In a clinical study by Cox et al., 10 children (aged 5-14 years) with post-resuscitation Glasgow Coma Scale scores of 5 to 8 were treated with bone marrow mononuclear cell transplantation. The group reported no adverse effects during the 6-months following transplantation. All patients were also evaluated with the PELOD (The Pediatric Logistic Organ Dysfunction) test, and no deleterious or reductive effects on white matter, gray substance or cerebrospinal fluid (CSF) were observed, even though these are commonly observed in $\mathrm{TBI}^{49}$.

MSCs are good candidates for therapies that focus primarily on preserving cells and host tissue, and do not require immunosuppressive treatment. They are able to differentiate and reach mature stages from local signs post-injury, stimulate the secretion of neurotrophic factors and decrease induced apoptosis. They can also maintain these effects for long periods due to their ability to survive. All of these factors are crucial in the activation of plasticity and local functional restoration.

The major challenge of cell transplantation in TBI is reaching the objectives of recovering motor functions, memory and cognition, as this depends directly on the region where cells are transplanted. For example, when implanted into the hippocampus, transplanted cells tend to survive longer and achieve a higher neuronal fate than when they are transplanted into different regions of the neocortex. It may also be possible to transplant different cells for different results, e.g., progenitor oligodendrocytes for remyelination in white matter, MSCs for neurotrophic support, and neural progenitors for cell replacement.

\section{SPINAL CORD INJURY}

Spinal cord injury (SCI) is a devastating condition of the CNS, with great physical and psychological repercussions. The disconnection of tracts and bundles of nerve fibers responsible for the ascendant and descendent communication of the spinal cord results in sensorimotor deficits, as well as loss of organ control in the body segments below the lesion. According to the National Spinal Cord Injury Statistical Center, approximately 12,000 new cases of SCI occur each year in the United States, affecting mostly men between the ages of 16 and 30. Despite the damage caused by the primary lesion to the spinal cord tissue, the pathophysiological mechanisms involved in the secondary phase (such as inflammatory, cytotoxic and vascular events and 
edema at the injury site) determine the extent of neurological deficits $^{50}$. Moreover, the formation of glial scars at the site of injury and the expression of several inhibitory molecules restricts fiber growth ${ }^{51}$. Considering the complex nature of SCIs, alternative treatments such as stem cell transplantation have been proposed as possible treatment options.

Olfactory ensheathing cells (OECs) are excellent candidates for cell transplantation. Other than myelinating axons and promoting axonal regeneration, there is strong evidence that OECs indirectly promote the endogenous capacity of Schwann cells to invade the injured site, thus creating a permissive microenvironment for axonal regeneration in the CNS. This interesting finding has motivated several clinical studies. One pilot study reported an improvement in overall (from A to C) and sensory and motor ASIA scores, changes in voluntary muscle control, bowel and bladder functions or sensation, and a reduction in the lesion cavity, as measured with MRI. Some adverse effects were reported: 1 patient had a lower ASIA score for the sensory component, and 2 other patients had increased trunk pain that lasted 2-3 months ${ }^{52}$.

In another study conducted by the same group, participants were 20 patients with complete motor injuries, AIS grades $\mathrm{A}$ and $\mathrm{B}$, all with chronic traumatic SCI (more the 1 year) and varying in age from 18-40 years old. The protocol the authors adopted emphasized an extensive preoperative rehabilitation program. Results showed improvements of 12 grades in AIS levels; only 1 patient had a lower AIS grade, and the group also reported improvements in FIM and walking index for $\mathrm{SCI}^{53}$.

Another clinical trial also suggested that promising results can be achieved with the combined transplantation of OECs and fragments of fetal spinal cord into the site of the lesion. Among all patients enrolled in the trial, 2 cases showed improvements in motor function and sensitivity, as well as overall control of the pelvic organs ${ }^{54}$.

Transplantation of mesenchymal stem cells (MSCs) in SCI animal models has been applied to promote sensorimotor and bladder function recovery via neural lineage differentiation, neurotrophic paracrine effects and post trauma inflammation regulation ${ }^{55}$. Some clinical trials have produced promising results: MSCs showed a great capacity to restore neurological deficits when administered intra-arterially or intravenously immediately following traumatic SCI. Syková et al. reported 1-year outcomes following transplantation of unmanipulated autologous bone marrow in 20 patients with complete SCI. Patients were treated both acutely (10-30 days after SCI; n=7) and chronically (2-17 months post-injury; $n=13$ ). When intra-arterial administration was used, $83 \%$ of patients reported neurological improvement within 3 months. The authors correlated neurological improvement with the therapeutic intervention, as $71 \%$ of acute patients showed neurological improvement while only $8 \%$ of chronic patients showed results ${ }^{56}$. In another study, Yoon and colleagues analyzed the timing of intervention in 35 patients with complete SCI (time since SCI: less than 14 days to more than 8 weeks) and noticed neurological recovery with earlier intervention: $30.4 \%$ in patients that were within 8 weeks of the injury, and none in patients more than 8 weeks post-injury ${ }^{57}$.

Two major conclusions can be made about MSCs: 1) it is safe and feasible to transplant MSCs via different routes; and 2) despite recent advancements, MSCs are still better at ensuring and stimulating local regeneration than properly differentiating into neurons ${ }^{58}$.

Another group of cells used for this kind of study are embryonic stem cells. All et al. transplanted oligodendrocyte progenitor cells (OPCs) derived from human ESC about 2 hours after the lesion. The OPCs were able to minimize the effects of the secondary injury, resulting in a partial restoration of ascending sensory pathways. The electrophysiological analyses showed an increase in latency by week 6 that was similar to the baseline analysis. The OPCs probably contributed to the remyelination of the spared axons ${ }^{59}$. Keirstead et al. transplanted OPCs at 7 days post-injury and noticed an improvement in axon remyelination and locomotor functions. However, when the cells were transplanted 10 months after injury, the group did not obtain the same results: there was cell survival and differentitation, but no remyelination or motor function improvement ${ }^{60}$. A major concern about the use of ESCs is tumor formation; when transplanted in the undifferentiated state, the remaining cells may form teratomas ${ }^{61}$.

\section{STEM CELLS IN ISCHEMIC STROKE}

In 2011, Lindvall and Kokaia ${ }^{27}$, published a review in which they discuss how far we are from using stem cells in stroke patients. In the paper, they describe some animal model studies in which functional recovery was obtained using NSCs derived from embryonic stem cells. The authors also reported functional recovery after transplantation of other non-neuronal cells, such as umbilical cord blood cells and cells from bone marrow. Although some studies have clearly produced positive results, the mechanisms behind these advances remain to be elucidated. Several hypotheses have been suggested, such as local increased energy metabolism or even a local change caused by the transplanted cells to stimulate endogenous repair. Failure to understand the mechanisms behind recovery may lead the scientific community to the erroneous belief that stem cell transplantation does not work. 


\section{STEM CELLS IN DEMYELINATING DISEASES}

Demyelinating diseases are pathological conditions in which there is a loss of myelin, sometimes due to repetitive episodes of small focal inflammatory reactions. These diseases include multiple sclerosis, transverse myelitis, Devic's disease, progressive multifocal leukoencephalopathy, optic neuritis and leukodystrophies. They may be idiopathic, genetic, autoimmune, or caused by infectious agents.

One possible solution is to transplant myelin-producing cells at the site of injury in order to repair the damage caused by inflammation. However, it is very difficult to cultivate and expand this particular type of cell, so one strategy is to transplant adult NPCs to differentiate into neurons, astrocytes and oligodendrocytes. Despite having critical characteristics of somatic cells, they may be subjected to larger expansions in vitro to become a renewable source of neural precursors and suitable for the treatment of various CNS disorders. In a model of experimental encephalomyelitis (EAE), adult NPCs showed high migration and directioning; they were found in greater numbers in places where there was demyelination, next to demyelinated cells or cells that had undergone remyelination. In addition to showing a capacity to differentiate into astrocytes, oligodendrocytes and neurons, they were also able to reduce glial scar formation. Adult NPCs seem to acquire a neurorestorative destination in vivo, i.e., when transplanted into an environment where neurodegeneration is predominant, they survive and acquire a functional mature phenotype and replenish damaged neural cells. However, when neuroinflammation is prevalent, they remain undifferentiated, retaining this phenotype and their ability to proliferate. This indicates that inflammation seems to be responsible for recruiting these cells and ensuring they remain in the affected areas for long periods. This occurs because NPCs have the same molecular pathway that lymphocytes patrolling the CNS, infiltrating CNS and producing gliogenic and neurogenic regulators; once the NPCs are in these niches, they exert neuroprotective effects that induce apoptosis of TH1 cells (proinflammatory) and spare TH2 cells (anti-inflammatory) ${ }^{62}$.

Despite the positive results reported above, there is still much to understand about the interaction of the transplanted cells with the host tissue, the best route of administration, and the safety and reliability of these transplants in humans, before we can safely move on to clinical studies.

\section{FINAL REMARKS}

Research on CNS restoration has seen important advances in recent years. This is particularly impressive in light of the fact that only 50 years ago, most people did not believe in neuroregeneration. With new discoveries revealing the potential of stem cells, cell therapy - which targets anatomical reconstruction and functional recovery has become a promising option for the treatment of neurological disorders. Work that improves our understanding of stem cell biology will help us move forward and identify, among other things, the best cell type to use. Results reported thus far suggest that an effective therapy will likely be available in a not so distant future.

\section{References}

1. Cajal SR. Estudios sobre la degeneración y regeneración del sistema nervioso. Vol. volume 1.1913, Madri: Moya

2. Hosseinkhani M, Shirazi R, Rajaei F, et al. Engineering of the embryonic and adult stem cell niches. Iran Red Crescent Med J 2013;15:83-92.

3. Bongso A, Richards M. History and perspective of stem cell research Best Pract Res Clin Obstet Gynaecol 2004;18:827-842.

4. Yamanaka S A. fresh look at iPS cells. Cell 2009;137:13-17.

5. Lander AD, Kimble J, Clevers H, et al. What does the concept of the stem cell niche really mean today? BMC Biol 2012;10:19

6. Inaba M, Yamashita YM. Asymmetric stem cell division: precision for robustness. Cell Stem Cell 2012;11:461-469.

7. McKay R. Stem cells in the central nervous system. Science 1997;276:66-71.

8. Gage FH. Mammalian neural stem cells. Science 2000;287:1433-1438.

9. Ming GL, Song H. Adult neurogenesis in the mammalian central nervous system. Annu Rev Neurosci 2005;28:223-250.

10. Gould E. How widespread is adult neurogenesis in mammals? Nat Rev Neurosci 2007;8:481-488.

11. Ming $G L$, Song $H$. Adult neurogenesis in the mammalian brain: significant answers and significant questions. Neuron 2011;70:687-702
12. Lepski G, Maciaczyk J, Jannes CE, et al. Delayed functional maturation of human neuronal progenitor cells in vitro. Mol Cell Neurosci 2011;47:36-44.

13. Wu CW, Chang YT, Yu L, et al. Exercise enhances the proliferation of neural stem cells and neurite growth and survival of neuronal progenitor cells in dentate gyrus of middle-aged mice. J Appl Physiol 2008;105:1585-1594.

14. Rochefort C, Gheusi G, Vincent JD, Lledo PM. Enriched odor exposure increases the number of newborn neurons in the adult olfactory bulb and improves odor memory. J Neurosci 2002;22:2679-2689.

15. Larsen CM, Grattan DR. Prolactin-induced mitogenesis in the subventricular zone of the maternal brain during early pregnancy is essential for normal postpartum behavioral responses in the mother. Endocrinology 2010;151:3805-3814.

16. Lledo PM, Alonso M, Grubb MS. Adult neurogenesis and functional plasticity in neuronal circuits. Nat Rev Neurosci 2006;7:179-193.

17. Kuhn HG, Winkler J, Kempermann G, Thal LJ, Gage FH. Epidermal growth factor and fibroblast growth factor-2 have different effects on neural progenitors in the adult rat brain. I Neurosci 1997;17:5820-5829.

18. Eisch AJ, Barrot M, Schad CA, Self DW, Nestler EJ. Opiates inhibit neurogenesis in the adult rat hippocampus. Proc Natl Acad Sci USA 2000;97:7579-7584 
19. Warner-Schmidt JL, Duman RS. Hippocampal neurogenesis: opposing effects of stress and antidepressant treatment. Hippocampus 2006;16:239-249.

20. Goetz CG. The history of Parkinson's disease: early clinical descriptions and neurological therapies. Cold Spring Harb Perspect Med 2011;1:a008862.

21. Michel PP, Toulorge D, Guerreiro S, Hirsch EC. Specific needs of dopamine neurons for stimulation in order to survive: implication for Parkinson disease. FASEB J 2013;27:3414-3423.

22. Freed CR, Greene PE, Breeze RE, et al. Transplantation of embryonic dopamine neurons for severe Parkinson's disease. N Engl J Med 2001;344:710-719.

23. Olanow CW, Goetz CG, Kordower JH, et al. A double-blind controlled trial of bilateral fetal nigral transplantation in Parkinson's disease. Ann Neurol 2003;54:403-414.

24. Winkler C, Kirik D, Bjorklund A. Cell transplantation in Parkinson's disease: how can we make it work? Trends Neurosci 2005;28:86-92.

25. Allan LE, Petit GH, Brundin P. Cell transplantation in Parkinson's disease: problems and perspectives. Curr Opin Neurol 2010;23:426-432.

26. Nishimura K, Takahashi J. Therapeutic application of stem cell technology toward the treatment of Parkinson's disease. Biol Pharm Bull 2013;36:171-175.

27. Lindvall O, Kokaia Z. Stem cell research in stroke: how far from the clinic? Stroke 2011;42:2369-2375.

28. Cicchetti F, Gross RE, Bulte JW, et al. Dual-modality in vivo monitoring of subventricular zone stem cell migration and metabolism. Contrast Media Mol Imaging 2007;2:130-138.

29. Bachoud-Levi AC. Neural grafts in Huntington's disease: viability after 10 years. Lancet Neurol 2009;8:979-981.

30. Capetian P, Knoth R, Maciaczyk J, et al. Histological findings on fetal striatal grafts in a Huntington's disease patient early after transplantation. Neuroscience 2009;160:661-675.

31. Lescaudron L, Naveilhan P, Neveu I. The use of stem cells in regenerative medicine for Parkinson's and Huntington's diseases. Curr Med Chem 2012;19:6018-6035.

32. Robberecht W, Philips T. The changing scene of amyotrophic lateral sclerosis. Nat Rev Neurosci 2013;14:248-264.

33. Svendsen CN, Langston JW. Stem cells for Parkinson disease and ALS: replacement or protection? Nat Med 2004;10:224-225.

34. Riley J, Federici T, Polak M, et al. Intraspinal stem cell transplantation in amyotrophic lateral sclerosis: a phase I safety trial, technical note, and lumbar safety outcomes. Neurosurgery 2012;71:405-416.

35. Freire MA. Pathophysiology of neurodegeneration following traumatic brain injury. West Indian Med J 2012;61:751-755.

36. Lim DA, Huang YC, Alvarez-Buylla A. The adult neural stem cell niche: lessons for future neural cell replacement strategies. Neurosurg Clin N Am 2007;18:81-92.

37. Shear DA, Tate MC, Archer DR, et al. Neural progenitor cell transplants promote long-term functional recovery after traumatic brain injury. Brain Res 2004;1026:11-22.

38. Lepski G, Jannes CE, Wessolleck J, Kobayashi E, Nikkhah G. Equivalent neurogenic potential of wild-type and GFP-labeled fetalderived neural progenitor cells before and after transplantation into the rodent hippocampus. Transplantation 2011;91:390-397.

39. Riess P, Zhang C, Saatman KE, et al. Transplanted neural stem cells survive, differentiate, and improve neurological motor function after experimental traumatic brain injury. Neurosurgery 2002;51:1043-1054.

40. Bakshi A, Shimizu S, Keck CA, et al. Neural progenitor cells engineered to secrete GDNF show enhanced survival, neuronal differentiation and improve cognitive function following traumatic brain injury. Eur J Neurosci 2006;23:2119-2134.
41. Hoane MR, Becerra GD, Shank JE, et al. Transplantation of neuronal and glial precursors dramatically improves sensorimotor function but not cognitive function in the traumatically injured brain. J Neurotrauma 2004;21:163-174.

42. Becerra GD, Tatko LM, Pak ES, Murashov AK, Hoane MR. Transplantation of GABAergic neurons but not astrocytes induces recovery of sensorimotor function in the traumatically injured brain. Behav Brain Res 2007;179:118-125.

43. Gao J, Prough DS, McAdoo DJ, et al. Transplantation of primed human fetal neural stem cells improves cognitive function in rats after traumatic brain injury. Exp Neurol 2006;201:281-292.

44. Gaillard A, Prestoz L, Dumartin B, et al. Reestablishment of damaged adult motor pathways by grafted embryonic cortical neurons. Nat Neurosci 2007;10:1294-1299.

45. Zhu J, Wu X, Zhang HL. Adult neural stem cell therapy: expansion in vitro, tracking in vivo and clinical transplantation. Curr Drug Targets 2005;6:97-110.

46. Zhu J, Zhou L, WingWu, F. Tracking neural stem cells in patients with brain trauma. N Engl J Med 2006;355:2376-2378.

47. Lu D, Li Y, Wang L, et al. Intraarterial administration of marrow stromal cells in a rat model of traumatic brain injury. J Neurotrauma 2001;18:813-819.

48. Mahmood A, Lu D, Chopp M. Intravenous administration of marrow stromal cells (MSCs) increases the expression of growth factors in rat brain after traumatic brain injury. J Neurotrauma 2004;21:33-39.

49. Cox CS, Jr. Baumgartner JE, Harting MT, et al. Autologous bone marrow mononuclear cell therapy for severe traumatic brain injury in children. Neurosurgery 2011;68:588-600.

50. Yip PK, Malaspina A. Spinal cord trauma and the molecular point of no return. Mol Neurodegener 2012;7:6.

51. Fawcett JW, Asher RA. The glial scar and central nervous system repair. Brain Res Bull 1999;49:377-3791.

52. Lima C, Pratas-Vital J, Escada P, et al. Olfactory mucosa autografts in human spinal cord injury: a pilot clinical study. J Spinal Cord Med 2006;29:191-206.

53. Lima C, Escada P, Pratas-Vital J, et al. Olfactory mucosal autografts and rehabilitation for chronic traumatic spinal cord injury. Neurorehabil Neural Repair 2010;24:10-22.

54. Rabinovich SS, Seledtsov VI, Poveschenko OV, et al, Transplantation treatment of spinal cord injury patients. Biomed Pharmacother 2003;57:428-433.

55. Osaka M, Honmou O, Murakami T, et al. Intravenous administration of mesenchymal stem cells derived from bone marrow after contusive spinal cord injury improves functional outcome. Brain Res 2010;1343:226-235

56. Sykova E, Homola A, Mazanec, R, et al. Autologous bone marrow transplantation in patients with subacute and chronic spinal cord injury. Cell Transplant 2006;15:675-687.

57. Yoon SH, Shim YS, Park YH, et al. Complete spinal cord injury treatment using autologous bone marrow cell transplantation and bone marrow stimulation with granulocyte macrophage-colony stimulating factor: Phase $1 / \|$ clinical trial. Stem Cells 2007;25:2066-2073.

58. Shin DA, Kim JM, Kim HI, et al. Comparison of functional and histological outcomes after intralesional, intracisternal, and intravenous transplantation of human bone marrow-derived mesenchymal stromal cells in a rat model of spinal cord injury. Acta Neurochir (Wien) 2013;155:1943-1950.

59. All AH, Bazley FA, Gupta S, et al. Human embryonic stem cellderived oligodendrocyte progenitors aid in functional recovery of sensory pathways following contusive spinal cord injury. PLoS One 2012;7:e47645. 
60. Keirstead HS, Nistor G, Bernal G, et al. Human embryonic stem cellderived oligodendrocyte progenitor cell transplants remyelinate and restore locomotion after spinal cord injury. J Neurosci 2005;25:4694-4705.

61. Matsuda R, Yoshikawa M, Kimura H, et al. Cotransplantation of mouse embryonic stem cells and bone marrow stromal cells following spinal cord injury suppresses tumor development. Cell Transplant 2009;18:39-54.

62. Pluchino S, Zanotti L, Rossi B, et al. Neurosphere-derived multipotent precursors promote neuroprotection by an immunomodulatory mechanism. Nature 2005;436:266-271. 